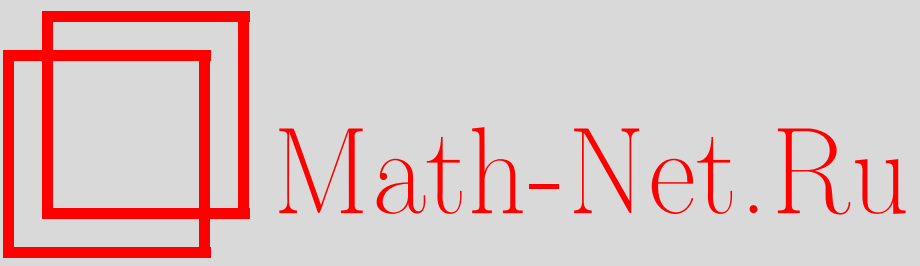

Э. А. Аринштейн, Критическая асимптотика корреляций в простой жидкости, ТМФ, 2004, том 138, номер 1, 127-138

DOI: https://doi.org/10.4213/tmf3

Использование Общероссийского математического портала Math-Net.Ru подразумевает, что вы прочитали и согласны с пользовательским соглашением

http://www . mathnet.ru/rus/agreement

Параметры загрузки:

IP : 54.84 .234 .179

26 апреля 2023 г., 10:37:42 
ТЕОРЕТИЧЕСКАЯ

И МАТЕМАТИЧЕСКАЯ

ФИЗИКА

Том 138, № 1

январь, 2004

(C) 2004 г.

Э. А. Аринштейн*

\section{КРИТИЧЕСКАЯ АСИМПТОТИКА КОРРЕЛЯЦИЙ В ПРОСТОЙ ЖИДКОСТИ}

Рассмотрено представление полной и прямой корреляционных функций жидкости через соответствующие спектральные плотности. Анализ свойств спектральных плотностей вскрывает механизм изменения аналитических свойств фурье-образа корреляционной функции и ее асимптотики в критической области. Показано, что известные ограничения на возможные значения показателя критической асимптотики следуют из этого механизма.

Ключевые слова: спектральная плотность, прямая корреляционная функция, критическая область, соотношение Орнштейна-Цернике.

\section{1. ВВЕДЕНИЕ}

Современная теория жидкости базируется на аппарате частичных функций распределения (частичных плотностей) [1], [2]. Для простых жидкостей с центральным бинарным взаимодействием унарная плотность является константой и достаточно найти бинарную корреляционную функцию $h(r)$, назьваемую также радиальной, либо полной, через которую выражаются давление, сжимаемость и внутренняя энергия. Утверждение о том, что через эту функцию выражаются все термодинамические величины, часто встречаюшееся в литературе, на наш взгляд, неточно. Так, для определения теплоемкости требуется найти тройную и четверную корреляции, через которые выражается производная от бинарной корреляции по температуре. Для бинарной корреляционной функции предложены приближенные уравнения, такие, например, как уравнение Перкуса-Йевика (ПЙ), гиперцепное уравнение (ГПЦ) и некоторые другие. Для вывода этих уравнений используется ряд качественных соображений, при этом наряду с полной корреляционной функцией в рассмотрение вводится прямая корреляционная функция Орнштейна-Цернике (ОЦ) $C(r)$, связанная в случае пространственно-однородной системы с полной корреляционной функцией $h(r)$ соотношением [1], [2]

$$
h\left(\vec{r}_{1}-\vec{r}_{2}\right)=C\left(\vec{r}_{1}-\vec{r}_{2}\right)+\rho \int h\left(\vec{r}_{1}-\vec{r}_{3}\right) C\left(\vec{r}_{3}-\vec{r}_{2}\right) d \vec{r}_{3}
$$

\footnotetext{
* Тюменский государственный университет, Тюмень, Россия. E-mail: earin@utmn.ru
} 
Погрешности, вносимые при переходе к отмеченным приближенным уравнениям, оценить довольно трудно. Тем не менее их решения, а также решения уравнений с некоторыми поправками находятся в качественном согласии с результатами численных расчетов в случае состояний, далеких от критического.

Соотношение ОЦ используется для анализа некоторых свойств корреляционных функций. Преобразование Фурье для функций $f(r)=\{h(r), C(r)\}$ имеет вид

$$
\tilde{f}(k)=\left(\frac{2}{\pi}\right)^{1 / 2} \int_{0}^{\infty} f(r) \frac{\sin (k r)}{k r} r^{2} d r .
$$

Соотношение ОЦ для фурье-образов этих функций

$$
\left[1+(2 \pi)^{3 / 2} \rho \tilde{h}(k)\right]\left[1-(2 \pi)^{3 / 2} \rho \widetilde{C}(k)\right]=1
$$

позволяет оценить асимптотику корреляционных функций в окрестности критической тОчки.

Так как функция $\sin (k r) /(k r)$ является четной аналитической функцией $k$, то можно предположить, что этим же свойством обладают и функции $\tilde{f}(k)$ и $\widetilde{C}(k)[2]$. Воспользуемся выражением для изотермической сжимаемости

$$
\frac{\rho \kappa_{T}}{\beta}=1+\rho \int h(r) d^{3} r=1+(2 \pi)^{3 / 2} \rho \tilde{f}(0)=\left[1-(2 \pi)^{3 / 2} \rho \tilde{C}(0)\right]^{-1} .
$$

Критическая точка определяется условием $\kappa_{T}=\infty$. Следовательно, в критической точке $1-\rho \int C(r) d^{3} r=1-(2 \pi)^{3 / 2} \rho \widetilde{C}(0)=0$, прямая корреляционная функция $C(r)$ остается “короткодействующей”, при $r \rightarrow \infty$ она убывает как $r^{-(3+\gamma)}, \gamma>0$, тогда как интеграл от полной корреляционной функции расходится на бесконечности вследствие возрастания радиуса корреляций. В окрестности критической точки выражение

$$
1-\rho \int C(r) d^{3} r=1-(2 \pi)^{3 / 2} \rho \widetilde{C}(0)
$$

мало. Разлагая $\sin (k r) /(k r)$ по степеням $k$ и используя очевидные обозначения, получим

$$
1-(2 \pi)^{3 / 2} \widetilde{C}(k) \approx 1-\rho \int C(r) d^{3} r+\frac{k^{2} \rho}{3 !} \int C(r) r^{2} d^{3} r=\left(\xi^{2}+k^{2}\right) R^{2} .
$$

Отсюда следует классическая асимптотика коррелящионной функции

$$
h(r) \approx \frac{e^{-\xi r}}{4 \pi \rho R^{2} r}
$$

причем в критической точке $\xi=0$ и $h(r) \sim r^{-1}$.

В то же время согласно современным представлениям [1]-[3] критическая асимптотика коррелящионной функции имеет иной вид,

$$
h(r) \sim e^{-\xi r} r^{-(1+\eta)}, \quad \eta>0,
$$

не совпадающий с (6) при $\eta \neq 0$. Хотя асимптотика $(7)$ (и соответственно $\tilde{h}(k) \sim k^{-2+\eta}$ ) не противоречит соотношению ОЦ, возникает вопрос о механизме вырождения асимптотики (6) в (7) и соответствующего изменения аналитических свойств функций $\tilde{h}(k)$ и $\widetilde{C}(k)$ в окрестности точки $k=0$ в критической области. Эта проблема может быть решена путем более аккуратного анализа уравнений для корреляционных функций и анализа спектральных плотностей функций $\tilde{h}(k)$ и $\widetilde{C}(k)$. 


\section{2. УРАВНЕНИЯ ДЛЯ КОРРЕЛЯЦИОННЫХ ФУНКЦИЙ, АСИМПТОТИЧЕСКИЕ ФОРМУЛЫ}

Корреляционные функции удовлетворяют бесконечной системе зацепляюшихся уравнений. Первое из этих уравнений - уравнение для полной бинарной корреляции - имеет вид [4], [5]

$$
\begin{aligned}
\ln (1 & +h(1,2))+\beta \phi(1,2)=\rho \int C(2,3) h(1,3) d(3)+ \\
& +\frac{\rho^{2}}{2} \int C_{3}(2,3,4) h(1,3) h(1,4) d(3,4)+ \\
& +\frac{\rho^{3}}{3 !} \int C_{4}(2,3,4,5) h(1,3) h(1,4) h(1,5) d(3,4,5)+\cdots .
\end{aligned}
$$

В этой формуле и далее цифрами $1,2,3, \ldots$ для краткости обозначены соответствующие радиус-векторы $\vec{r}_{1}, \vec{r}_{2}, \vec{r}_{3}, \ldots$ В формуле $(8) C(2,3) \equiv C_{2}(2,3)$ обозначает прямую корреляционную функцию ОЦ,

$$
C_{n}(2,3, \ldots, n+1)=\frac{\delta^{n-2} C_{2}(2,3)}{\delta \rho(4) \ldots \delta \rho(n+1)}
$$

- старшие прямые корреляции, они являются функциональными производными от $C(2,3)$ по флуктуирующей унарной плотности и связаны с соответствующими полными корреляциями интегральными соотношениями

$$
\begin{aligned}
C_{3}(1,2,3)= & \int\left[h_{3}(4,5,6)-h_{2}(4,5) h_{2}(4,6)-h_{2}(4,5) h_{2}(5,6)-h_{2}(4,6) h_{2}(5,6)\right] \times \\
& \times \prod\left[\delta(i, i+3)-\rho C_{2}(i, i+3)\right] d(4,5,6)-\rho \int C_{2}(1,4) C_{2}(2,4) C_{2}(3,4) d(4)
\end{aligned}
$$

и аналогично для $C_{n}$ при $n>3$. Уравнение (8) можно рассматривать как замкнутое уравнение для $h(r)$, если уравнения для старших корреляций разрешимы хотя бы в виде интегростепенных рядов по $h_{2}$.

В частности, полагая $C_{n}=0, n \geqslant 3$, получим ГПЦ-приближение, полагая

$$
C_{n+1}(\{n+1\})=(n-1) ! \prod_{i=2}^{n+1} C_{2}(1, i),
$$

получим приближение ПЙ. Установить из общих соображений точность этих приближений невозможно. Можно получить связь функций $C_{n}$ с термодинамическими величинами. Дифференцируя выражение для обратной величины изотермической сжимаемости (4), получим

$$
\begin{aligned}
\frac{\partial^{n} \beta p}{\partial \rho^{n}}= & \frac{\partial^{n-1}}{\partial \rho^{n-1}} \frac{\beta}{\rho \kappa_{T}}=-\rho \int \frac{\delta^{n-1} C_{2}(1,2)}{\delta \rho(3) \ldots \delta \rho(n+1)} d(2, \ldots, n+1)- \\
& -(n-1) \int \frac{\delta^{n-2} C_{2}(1,2)}{\delta \rho(3) \ldots \delta \rho(n)} d(2, \ldots, n)= \\
= & -\rho \int C_{n+1}(1,2, \ldots, n+1) d(2, \ldots, n+1)- \\
& -(n-1) \int C_{n}(1,2, \ldots, n) d(2, \ldots, n) .
\end{aligned}
$$

5 Теоретическая и математическая физика, т. 138, № 1, 2004 г. 
При $n=2$ выражение (9) сводится к равенству

$$
\rho \frac{\partial^{2} \beta p}{\partial \rho^{2}}=\rho \frac{\partial}{\partial \rho} \frac{\beta}{\rho \kappa_{T}}=1-\rho \int C_{2}(1,2) d(2)-\left(1+\rho^{2} \int C_{3}(1,2,3) d(2,3)\right) .
$$

При температурах выше критической сушествует линия закритических переходов, где обратная сжимаемость имеет минимум. Критическая точка также принадлежит этой линии, следовательно, в критической точке не только

$$
1-\rho \int C_{2}(1,2) d(2)=0
$$

но и

$$
1+\rho^{2} \int C_{3}(1,2,3) d(2,3)=0
$$

т.е. не только функция $C_{2}$, но и $C_{3}$ остаются быстроубывающими в критической точке и интеграл от этих функций сходится.

Разлагая логарифм в левой части уравнения (8) по степеням $h_{2}$ и используя соотношение ОЦ, приведем это уравнение к форме

$$
\begin{aligned}
C_{2}(1,2)= & -\phi(1,2)+\frac{1}{2}\left(h_{2}^{2}(1,2)+\rho^{2} \int C_{3}(2,3,4) h_{2}(1,3) h_{2}(1,4) d(3,4)\right)- \\
& -\frac{1}{3}\left(h_{2}^{3}(1,2)-\frac{\rho^{3}}{2} \int C_{4}(2,3,4,5) \prod h_{2}(1, i) d(3,4,5,)\right)+\cdots
\end{aligned}
$$

определяющей разложение прямой корреляции ОЦ в интегростепенной ряд по $h_{2}$. Совместно с соотношением ОЦ это уравнение определяет $h_{2}$. Из него видно, что асимптотически, на расстояниях, где потенциал $\phi(1,2)=0$, функция $C_{2} \sim h_{2}^{2}$, но в критической точке этот член асимптотики исчезает. Учет старших членов разложения (8) не меняет этот вывод. Действительно, в силу симметрии координат (1) и (2) и с учетом того, что две функции $C_{3}$ могут быть соединены двумя функциями $h_{2}$, из полного разложения могут быть выделены дополнительно члены

$$
\begin{aligned}
& \frac{1}{2}\left[\rho^{2} \int C_{3}(1,3,4) h_{2}(2,3) h_{2}(2,4) d(3,4)+\right. \\
& \left.\quad+\rho^{4} \int C_{3}(1,3,4) C_{3}(2,5,6) h_{2}(3,5) h_{2}(4,6) d(3,4,5,6)\right]
\end{aligned}
$$

Главный асимптотический член равен

$$
\frac{1}{2}\left(1+\rho^{2} \int C_{3}(3,4,5) d(3,4,5)\right)^{2} h_{2}^{2}(1,2) .
$$

В критической области он имеет даже более высокий, второй порядок малости.

Отметим, что в ГПЦ-приближении указанной компенсации квадратичного члена асимптотики не происходит, в приближении Пй $C_{2}(r)=0$ при $\phi(r)=0$. Другими словами, оба эти приближения дают неадекватное описание критической точки. Ясно, что из 
общих соображений, учитывая необходимость перегруппировки членов ряда (11), определить характер поведения функций $C_{n}, n>3$, и других членов этого ряда, симметричных выписанным, довольно сложно. Тем более сложно указать условия интегрируемости этих функций, значения интегралов от них и условия обращения в нуль соответствующих членов асимптотического разложения функции $C_{2}$. Тем не менее можно принять гипотезу, что $C_{2} \sim h_{2}^{n}$, где $n>2$ - показатель критической изотермы, не обязательно целый. В этом случае для него используется обозначение $\delta$ [1]-[3].

Асимптотика полной корреляционной функции $h(r)$ определяется некоторыми свойствами прямой корреляционной функции ОЦ. Представим полную корреляцию в виде [6], $[7]$

$$
h(r)=a \frac{e^{-\xi r}}{\xi r}+\frac{h^{\prime}(r)}{r}, \quad h^{\prime}=o\left(\frac{e^{-\xi r}}{\xi}\right) .
$$

При подстановке (12) в соотношение ОЦ получаем

$$
\begin{aligned}
& a \frac{e^{-\xi r}}{\xi r}\left(1-4 \pi \rho \int_{0}^{\infty} C\left(r^{\prime}\right) \frac{\operatorname{sh}\left(\xi r^{\prime}\right)}{\xi r^{\prime}} r^{\prime 2} d r^{\prime}\right)+\frac{h^{\prime}(r)}{r}-\rho \int C\left(r_{13}\right) \frac{h^{\prime}\left(r_{23}\right)}{r_{23}} d(3)= \\
& =C(r)-\frac{4 \pi \rho a}{r} \int_{r}^{\infty} C\left(r^{\prime}\right) \frac{\operatorname{sh}\left(\xi\left(r^{\prime}-r\right)\right)}{\xi^{2}} r^{\prime} d r^{\prime}
\end{aligned}
$$

Главный член, содержащий $a e^{-\xi r} /(\xi r)$, исчезает, если определить $\xi$ из уравнения

$$
\begin{aligned}
1- & 4 \pi \rho \int_{0}^{\infty} C(r) \frac{\operatorname{sh}(\xi r)}{\xi r} r^{2} d r=1-\rho \int C(1,2) d(2)+ \\
& +4 \pi \rho \int_{0}^{\infty} C(r)\left(\frac{\operatorname{sh}(\xi r)}{\xi r}-1\right) r^{2} d r=0
\end{aligned}
$$

(точнее, если определить $\xi$ как решение этого уравнения с наименьшей положительной действительной частью).

Умножая уравнение (13) на $r \operatorname{sh}(\xi r)$ и интегрируя, получим слева

$$
\int_{0}^{\infty} h(r) \operatorname{sh}(\xi r) d r\left(1-4 \pi \rho \int_{0}^{\infty} C(r) \frac{\operatorname{sh}(\xi r)}{\xi r} r^{2} d r\right)=0
$$

и окончательно

$$
\frac{\xi^{3}}{4 a \pi \rho}=4 \pi \rho \int_{0}^{\infty} C(r)\left(\operatorname{ch}(\xi r)-\frac{\operatorname{sh}(\xi r)}{\xi r}\right) r^{2} d r .
$$

Уравнения (14), (15) определяют радиус $1 / \xi$ главного асимптотического члена корреляционной функции $h(r)$ и его амплитуду $a$. Из конечности правой части уравнения (15) следует, что эта амплитуда в критической точке обращается в нуль. Ряд свойств остающейся неклассической асимптотики можно выяснить на основе анализа спектральной плотности корреляционных функций. 


\section{3. СПЕКТРАЛЬНЫЕ ПЛОТНОСТИ, МЕХАНИЗМ ВОЗНИКНОВЕНИЯ НЕКЛАССИЧЕСКОЙ АСИМПТОТИКИ}

Для исследования аналитических свойств функций $\tilde{h}(k)$ и $\widetilde{C}(k)$ необходимо перейти в комплексную плоскость переменной $k$ и наряду с фурье-преобразованием функций $h(r)$ и $C(r)$ рассмотреть их представление в виде интеграла Лапласа,

$$
f(r)=\int_{0}^{\infty} \bar{f}(t) \frac{e^{-t r}}{t r} d t .
$$

Замена функции $e^{-t r}$ на $e^{-t r} /(t r)$ обусловлена тем, что именно последняя функция осушествляет представление группы движений трехмерного пространства [8], в частности, именно такой вид имеет асимптотика (6). Эта функция удовлетворяет теореме сложения

$$
\varphi_{0}\left(t\left|\vec{r}_{1}-\vec{r}_{2}\right|\right)=\sum(-1)^{n}(2 n+1) P_{n}(\cos \theta) \varphi_{n}\left(t r_{1}\right) \psi_{n}\left(r_{2}\right)
$$

где

$$
\varphi_{n}(x)=x^{n}\left(\frac{1}{x} \frac{d}{d x}\right)^{n} \frac{\operatorname{sh} x}{x}, \quad \psi_{n}(x)=x^{n}\left(\frac{1}{x} \frac{d}{d x}\right)^{n} \frac{e^{-x}}{x}, \quad r_{1}<r_{2} .
$$

Представление (16) определяет особые точки фурье-образа функции $\tilde{f}(k)$,

$$
\tilde{f}(k)=\sqrt{\frac{2}{\pi}} \frac{1}{2 i k} \int_{0}^{\infty}\left(\frac{1}{t-i k}-\frac{1}{t+i k}\right) \bar{f}(t) \frac{d t}{t}=\sqrt{\frac{2}{\pi}} \int_{0}^{\infty} \frac{\bar{f}(t) d t}{\left(t^{2}+k^{2}\right) t},
$$

т.е. функция $\bar{f}(t)$ является спектральной плотностью для $\tilde{f}(k)$.

Непосредственным интегрированием, либо используя теорему сложения (17), получим

$$
\int e^{-t r_{13}-t^{\prime} r_{23}} \frac{d(3)}{t r_{13} t^{\prime} r_{23}}=\frac{4 \pi}{t t^{\prime}} \frac{e^{-t r}-e^{-t^{\prime} r}}{t^{\prime 2}-t^{2}} .
$$

При подстановке в соотношение ОЦ это дает

$$
\begin{gathered}
\int_{0}^{\infty} \frac{e^{-t r}}{t r}[\bar{h}(t)-\bar{C}(t)] d t=4 \pi \rho \int_{0}^{\infty} \int_{0}^{\infty} \bar{h}\left(t^{\prime}\right) \bar{C}(t) \frac{e^{-t r}-e^{-t^{\prime} r}}{\left(t^{\prime 2}-t^{2}\right) t t^{\prime} r} d t d t^{\prime}= \\
\quad=4 \pi \rho \int_{0}^{\infty} \frac{e^{-t r}}{t r} d t \int_{0}^{\infty} P \frac{\bar{h}(t) \bar{C}\left(t^{\prime}\right)+\bar{h}\left(t^{\prime}\right) \bar{C}(t)}{\left(t^{\prime 2}-t^{2}\right) t^{\prime}} d t^{\prime}
\end{gathered}
$$

( $P$ - символ интеграла в смысле главного значения по Коши), откуда в силу полноты системы функций $\psi_{0}(x)$ на полуоси получим

$$
\bar{h}(t)\left(1-4 \pi \rho \int_{0}^{\infty} P \frac{\bar{C}\left(t^{\prime}\right) d t^{\prime}}{\left(t^{\prime 2}-t^{2}\right) t^{\prime}}\right)=\bar{C}(t)\left(1+4 \pi \rho \int_{0}^{\infty} P \frac{\bar{h}\left(t^{\prime}\right) d t^{\prime}}{\left(t^{\prime 2}-t^{2}\right) t^{\prime}}\right) .
$$

Спектр функции $\tilde{h}(k)$ имеет пару симметричных изолированных точек $\pm i \xi$, определяюших главньй асимптотический член. Разлагая $f(r)$ по степеням функции $e^{-\xi r} /(\xi r)$ и учитывая соотношения

$$
\frac{e^{-2 \xi r}}{r^{2}}=\int_{2 \xi}^{\infty} \frac{e^{-t r}}{r} d t, \quad \frac{e^{-3 \xi r}}{r^{3}}=\int_{3 \xi}^{\infty} \frac{e^{-t r}}{r}(t-3 \xi) d t
$$


(и аналогичные для более высоких степеней), приходим к выводу, что спектр функций $\tilde{h}(k)$ и $\widetilde{C}(k)$ кроме изолированных точек содержит на мнимой оси кусочно-гладкие (с шагом порядка $\xi$ ) части, начинаюшиеся с точек $\pm 2 i \xi$ (в общем случае - с точек $\pm n i \xi$ ).

В критической области изолированный полюс спектральной плотности функции $h$ исчезает в соответствии с уравнением (15). Остается непрерывный спектр, начинающийся почти с нуля и практически гладкий, что приводит к изменению аналитического характера этой функции, так как начало спектра является не полюсом, а точкой ветвления. Новый характер поведения функции $h$ охватывает область $r_{0} \ll r \ll \xi^{-1}$, где $r_{0}$ радиус ближних коррелящий, имеющий порядок радиуса действия сил, и возникает при $r_{0} \ll \xi^{-1}$, т.е. только в критической области. Точка ветвления спектра изменяет аналитические свойства и прямой корреляционной функции, связанной с полной соотношением ОЦ. Это приводит к тому, что возможен нецельй показатель степени критической изотермы $\delta$.

При исследовании критической асимптотики в области $r_{0} \ll r \ll \xi^{-1}$ можно опустить обрашающийся в нуль член, определяюший классическую асимптотику. При этом в формуле (16) в случаях, когда подынтегральная функция не стремится на нижнем пределе к нулю, следует заменить нижний предел интегрирования на $n \xi$.

\section{4. ДВУМЕРНЫЙ СЛУЧАЙ}

В двумерном случае изложенный подход требует некоторой модификации.

Преобразование Фурье имеет вид

$$
f(r)=2 \pi \int_{0}^{\infty} J_{0}(k r) \tilde{f}(k) k d k
$$

где $J_{0}$ - функция Бесселя. Соотношение ОЦ, выражения для обратной сжимаемости и ее производной по плотности имеют тот же вид, что и в трехмерном случае. Необходимо лишь заменить коэффициент $(2 \pi)^{3 / 2}$ перед фурье-компонентами коэффициентом $2 \pi$.

Представление группы движений в двумерном случае осушествляют функции Макдональда $K_{0}(t r)$ [8], удовлетворяющие теореме сложения,

$$
K_{0}\left(t\left|\vec{r}_{1}-\vec{r}_{2}\right|\right)=\sum e^{i n \theta} I_{n}\left(t r_{1}\right) K_{n}\left(t r_{2}\right), \quad r_{1}<r_{2}
$$

Вместо интеграла Лапласа используется представление

$$
f(r)=\int_{0}^{\infty} K_{0}(t r) \bar{f}(t) d t
$$

Действительно, выражая трехмерное расстояние через двумерное и усредняя по третьей координате $z$, получим

$$
\int_{0}^{\infty} e^{-t \sqrt{r^{2}+z^{2}}} \frac{d z}{\sqrt{r^{2}+z^{2}}}=\int_{0}^{\infty} e^{-t r \operatorname{ch} u} d u=K_{0}(t r)
$$


Так как $\int_{0}^{\infty} K_{0}(t r) J_{0}(k r) r d r=1 /\left(t^{2}+k^{2}\right)$, то из (24) следует

$$
\tilde{f}(k)=\int_{0}^{\infty} \frac{\bar{f}(t) d t}{t^{2}+k^{2}},
$$

т.е. и в этом случае функция $\bar{f}(t)$ является спектральной плотностью для функции $\tilde{f}(k)$.

Для определения радиуса и амплитуды главного члена асимптотики функции $h(r)$ представим ее в виде

$$
f(r)=a K_{0}(\xi r)+h^{\prime}(r),
$$

что дает уравнения для обратного радиуса корреляции $\xi$ и амплитуды главного асимптотического члена,

$$
\begin{gathered}
1-\rho \int C(1,2) d(2)+2 \pi \rho \int_{0}^{\infty} C(r)\left(I_{0}(\xi r)-1\right) r d r=0 \\
\frac{\xi^{2}}{a \pi \rho}=2 \pi \rho \int_{0}^{\infty} C(r) \xi r I_{1}(\xi r) r d r
\end{gathered}
$$

аналогичные уравнениям (14), (15). Из уравнения (28), так же как из (15), следует исчезновение в критической точке члена, определяюшего классическую асимптотику.

При использовании представления (24), теоремы сложения и интеграла (25) соотношение ОЦ принимает в двумерном случае вид

$$
\begin{aligned}
\int_{0}^{\infty} \frac{(\bar{h}(t)-\bar{C}(t)) d t}{t^{2}+k^{2}} & =2 \pi \rho \int_{0}^{\infty} \int_{0}^{\infty} \frac{\bar{h}(t) \bar{C}(t)}{\left(t^{2}+k^{2}\right)\left(t^{\prime 2}+k^{2}\right)} d t d t^{\prime}= \\
& =2 \pi \rho \int_{0}^{\infty} \frac{d t}{t^{2}+k^{2}} \int_{0}^{\infty} P \frac{\bar{h}(t) \bar{C}\left(t^{\prime}\right)+\bar{h}\left(t^{\prime}\right) \bar{C}(t)}{t^{\prime 2}-t^{2}} d t^{\prime}
\end{aligned}
$$

откуда в силу полноты на полуоси системы функций $K_{0}(t x)$, приводящей к появлению в обеих частях равенства (29) знаменателя $t^{2}+k^{2}$, получим

$$
\bar{h}(t)\left(1-2 \pi \rho \int_{0}^{\infty} P \frac{\bar{C}\left(t^{\prime}\right) d t^{\prime}}{t^{\prime 2}-t^{2}}\right)=\bar{C}(t)\left(1+2 \pi \rho \int_{0}^{\infty} P \frac{\bar{h}\left(t^{\prime}\right) d t^{\prime}}{t^{\prime 2}-t^{2}}\right) .
$$

Это соотношение аналогично соотношению (20) для трехмерного случая.

Сушествование кусочно-гладкой части спектра следует из формул [9]

$$
\begin{gathered}
K_{0}^{2}(\xi r)=2 \int_{2 \xi}^{\infty} K_{0}(t r) \frac{d t}{\sqrt{t^{2}-4 \xi^{2}}}, \\
K_{0}^{3}(\xi r)=8 \int_{3 \xi}^{\infty} K_{0}(t r) \frac{d t}{t} \int_{0}^{\operatorname{arch} \frac{t-\xi}{2 \xi}} \frac{d \tau}{\sqrt{\left(1-\left(\frac{2 \operatorname{ch} \tau+1}{t} \xi\right)^{2}\right)\left(1-\left(\frac{2 \operatorname{ch} \tau-1}{t} \xi\right)^{2}\right)}}, \ldots,
\end{gathered}
$$

являюшихся аналогом и результатом интегрирования формул (21) по третьей координате. Отсюда следует, как и в трехмерном случае, изменение аналитического характера асимптотики в критической области. 


\section{5. СТЕПЕННАЯ АСИМПТОТИКА}

Рассмотрим возможность возникновения неклассической степенной асимптотики радиальной функции и ограничения на значение показателей этой асимптотики. Такая асимптотика существует на расстояниях $r_{0} \ll r \ll \xi^{-1}$, волновые числа фурье-образов корреляционных функций и параметры спектральных плотностей ограничены условиями $\xi \ll k \ll r_{0}^{-1}, \xi \ll t \ll r_{0}^{-1}$. Таким образом, последуюший анализ относится исключительно к окрестности критического состояния. При этом следует иметь в виду, что на спектральные плотности налагаются ограничения, которые требуют изменения их характера вне указанного интервала. Так как функции $f(r)=\{h(r), C(r)\}$ конечны при $r=0$ и $f(0)=\int_{0}^{\infty} \bar{f}(t) d t /(r t)$, то интегралы $\int_{0}^{\infty} \bar{f}(t) d t / t=0$ и $\int_{0}^{\infty} \bar{f}(t) d t=-f(0)$ сходятся как на нижнем, так и на верхнем пределах. Другие свойства интегралов от спектральных плотностей следуют из выражения (18) для фурье-образов $\tilde{f}(k)$ функций $f(r)$. В критической области

$$
1-4 \pi \rho \int_{0}^{\infty} \bar{C}(t) \frac{d t}{t^{3}}=1-(2 \pi)^{3 / 2} \widetilde{C}(0) \approx 0, \quad 4 \pi \rho \int_{0}^{\infty} \bar{h}(t) \frac{d t}{t^{3}} \gg 1,
$$

т.е. последний интеграл расходится на нижнем пределе.

С учетом того, что

$$
\begin{gathered}
1-4 \pi \rho \int_{0}^{\infty} P \frac{\bar{C}\left(t^{\prime}\right) d t^{\prime}}{\left(t^{\prime 2}-t^{2}\right) t^{\prime}}=1-4 \pi \rho \int_{0}^{\infty} P \frac{\bar{C}\left(t^{\prime}\right)\left(t^{\prime 2}-t^{2}+t^{2}\right) d t^{\prime}}{\left(t^{\prime 2}-t^{2}\right) t^{\prime 3}}= \\
=1-(2 \pi)^{3 / 2} \widetilde{C}(0)-4 \pi \rho t^{2} \int_{0}^{\infty} P \frac{\bar{C}\left(t^{\prime}\right) d t^{\prime}}{\left(t^{\prime 2}-t^{2}\right) t^{\prime 3}} \approx-4 \pi \rho t^{2} \int_{0}^{\infty} P \frac{\bar{C}\left(t^{\prime}\right) d t^{\prime}}{\left(t^{\prime 2}-t^{2}\right) t^{\prime 3}} \\
1+4 \pi \rho \int_{0}^{\infty} P \frac{\bar{h}\left(t^{\prime}\right) d t^{\prime}}{\left(t^{\prime 2}-t^{2}\right) t^{\prime}} \approx 4 \pi \rho \int_{0}^{\infty} P \frac{\bar{h}\left(t^{\prime}\right) d t^{\prime}}{\left(t^{\prime 2}-t^{2}\right) t^{\prime}}
\end{gathered}
$$

соотношение (20) в критической области приводится к виду

$$
-\bar{h}(t) t^{2} \int_{0}^{\infty} P \frac{\bar{C}\left(t^{\prime}\right) d t^{\prime}}{\left(t^{\prime 2}-t^{2}\right) t^{\prime 3}}=\bar{C}(t) \int_{0}^{\infty} P \frac{\bar{h}\left(t^{\prime}\right) d t^{\prime}}{\left(t^{\prime 2}-t^{2}\right) t^{\prime}}
$$

Из степенного поведения функций $h(r)=A / r^{1+\eta}, C(r)=B / r^{3+\gamma}$ следует, что $\bar{h}(t)=$ $A t^{\eta} / \Gamma(\eta), \bar{C}(t)=B t^{2+\gamma} / \Gamma(2+\gamma)$. При подстановке последних равенств в соотношение $(32)$ получаем

$$
-\int_{0}^{\infty} P \frac{t^{\gamma-1} d t}{t^{2}-1}=\int_{0}^{\infty} P \frac{t^{\eta-1} d t}{t^{2}-1} \equiv-\int_{0}^{\infty} P \frac{t^{-\eta+1} d t}{t^{2}-1}
$$

откуда следует связь показателей $\eta+\gamma=2\left(\right.$ так как $\int_{0}^{\infty} P t^{\gamma-1} d t /\left(1-t^{2}\right)=(\pi / 2) \times$ $\operatorname{ctg}(\pi \gamma / 2)$, то можно записать эту связь в виде $\eta+\gamma=2+2 n$. Однако значения $n \neq 0$ приводят к расходимости одного либо обоих интегралов и должны быть исключены). 
При исследовании соотношения ОЦ (3) для фурье-образов функций $h$ и $C$ в критической области следует учесть, что

$$
\begin{gathered}
(2 \pi)^{3 / 2} \rho \tilde{h}(k)=4 \pi \rho \int_{0}^{\infty} \frac{\bar{h}(t) d t}{\left(t^{2}+k^{2}\right) t} \gg 1, \\
1-(2 \pi)^{3 / 2} \rho \widetilde{C}(k)=1-4 \pi \rho \int_{0}^{\infty} \frac{\bar{C}(t)\left(t^{2}+k^{2}-k^{2}\right) d t}{\left(t^{2}+k^{2}\right) t^{3}}= \\
=1-(2 \pi)^{3 / 2} \rho \widetilde{C}(0)+4 \pi \rho k^{2} \int_{0}^{\infty} \frac{\bar{C}(t) d t}{\left(t^{2}+k^{2}\right) t^{3}} \approx 4 \pi \rho k^{2} \int_{0}^{\infty} \frac{\bar{C}(t) d t}{\left(t^{2}+k^{2}\right) t^{3}} .
\end{gathered}
$$

Используя аппроксимацию $\bar{h}(t)=A t^{\eta} / \Gamma(\eta), \bar{C}(t)=B t^{2+\gamma} / \Gamma(2+\gamma)$ и значение интеграла $\int_{0}^{\infty} t^{\eta-1} d t /\left(1+t^{2}\right)=\pi /\left(2 \sin \frac{\pi \eta}{2}\right)$, приведем соотношение (3) к виду

$$
\frac{4 \pi^{4} A B \rho^{2} k^{\eta+\gamma-2}}{\Gamma(\eta) \Gamma(\gamma+2) \sin \frac{\pi \eta}{2} \sin \frac{\pi \gamma}{2}} \approx 1
$$

откуда вновь следует связь $\eta+\gamma=2$.

Из гипотезы о степенной связи прямой и полной корреляций $C(r) \sim(h(r))^{\delta}$ следует, что $(1+\eta) \delta=3+\gamma$. Отсюда окончательно получаем, что

$$
\eta=\frac{6}{\delta+1}-1, \quad \gamma=3-\frac{6}{\delta+1} .
$$

Найденные выражения (35) совпадают с известными [1]-[3].

Из интегрируемости функции $C(r)$ следует, что $\gamma>0$ и $\delta>2$. Из условия $\eta>0$ следует $\delta<5$. При $\delta=5$ интеграл (16) расходится на нижнем пределе, этот предел должен быть обрезан на величине порядка $n \xi$, что приводит к неклассической асимптотике

$$
h(r)=A \int_{n \xi}^{\infty} e^{-t r} \frac{d t}{t r}=\frac{A}{r} \operatorname{Ei}(-n \xi r)
$$

практически совпадаюшей с асимптотикой (7) при малых $\eta$. Двумерный случай исследуется аналогично. Условия интегрируемости спектральной плотности и ограничение на значение интеграла от нее следуют из того, что при малых $x$ функция Макдональда $K(x) \approx \ln (1 / x)$. Неклассическая асимптотика $h(r) \sim r^{\eta}, C(r) \sim r^{2+\gamma}$ определяет поведение спектральной плотности в критической области: $\bar{h}(t) \sim 2^{2-\eta} t^{\eta-1} / \Gamma^{2}(\eta / 2)$ и аналогично для $C(r)$.

Дальнейший анализ проводится практически таким же образом и приводит к той же связи показателей $\eta+\gamma=2$. Из гипотезы о степенной связи прямой и полной корреляционных функций следует, что $2+\gamma=\eta \delta$ и

$$
\eta=\frac{4}{\delta+1}, \quad \gamma=2 \frac{\delta-1}{\delta+1} .
$$




\section{ПРИЛОЖЕНИЕ}

Выражения для старших прямых корреляций $C_{n}$ можно получить, используя метод производяшего функционала [4]. Зависяшая от изменений дополнительного внешнего поля плотность

$$
\rho(1)=\frac{(1+u(1)) \delta W(u)}{\delta u(1)} \equiv(1+u(1)) W_{1}(1),
$$

где $W=\ln L(u)$ и $L(u)$ - производяший функционал частичных плотностей, введенный Боголюбовым. Функциональное касательное преобразование

$$
\widetilde{W}=W+\int\left[\rho(q) \ln \frac{\rho(q)}{1+u(q)}-\rho(q)-\rho^{0}(q) \ln \rho^{0}(q)+\rho^{0}(q)\right] d q,
$$

где $\rho^{0}$ - фиксированная, неварьируемая плотность, определяет систему функций $C_{n}(1, \ldots, n) \equiv \widetilde{W}_{n}(1, \ldots, n) \equiv \delta^{n} \widetilde{W} /(\delta \rho(1) \ldots \delta \rho(n))$. Из выражения (П.1) имеем

$$
\begin{gathered}
\widetilde{W}_{1}(1)=\ln [\rho(1) /(1+u(1))]=\ln W_{1}(1), \\
\widetilde{W}_{2}(1,2)=\frac{\delta(1,2)}{\rho(1)}-\frac{\delta u(1)}{(1+u(1)) \delta \rho(1)}=\int \frac{W_{2}(1, q)}{W_{1}(1) W_{1}(q)} \frac{\rho(q)}{1+u(q)} \frac{\delta u(q)}{\delta \rho(2)} d q .
\end{gathered}
$$

Так как $W_{2}(1,2) /\left(W_{1}(1) W_{1}(2)\right)=h_{2}(1,2)$, то

$$
C_{2}(1,2)=\int h_{2}(1, q)\left[\delta(2, q)-C_{2}(2, q) \rho(q)\right] d q,
$$

т.е. $C_{2}$ удовлетворяет соотношению ОЦ и функции $C_{n}$ являются прямыми корреляционными функциями.

Выражение для $C_{3}$ получим, варьируя соотношение (П.2),

$$
\begin{gathered}
\int C_{3}(1,3, q)\left[\delta(2, q)+h_{2}(2, q) \rho(q)\right] d q+C_{2}(1,3) h_{2}(2,3)= \\
=\int \frac{\delta h_{2}(2, q)}{\delta \rho(3)}\left[\delta(1, q)-C_{2}(1, q) \rho(q)\right] d q .
\end{gathered}
$$

Умножим это выражение на $\left[\delta\left(2,2^{\prime}\right)-C_{2}\left(2,2^{\prime}\right) \rho(2)\right] d(2)$. Взяв интеграл по $d(2)$, с учетом соотношения ОЦ и того, что

$$
\begin{aligned}
\frac{\delta h_{2}(2, q)}{\delta \rho(3)}= & \int \frac{\delta h_{2}(2, q)}{\delta u\left(q^{\prime}\right)} \frac{\delta u\left(q^{\prime}\right)}{\delta \rho(3)} d q^{\prime}=\int\left[h_{3}\left(2, q, q^{\prime}\right)-\right. \\
& \left.-h_{2}\left(2, q^{\prime}\right) h_{2}(2, q)-h_{2}\left(q, q^{\prime}\right) h_{2}(2, q)\right]\left[\delta\left(q^{\prime}, 3\right)-C_{2}\left(q^{\prime}, 3\right) \rho\left(q^{\prime}\right)\right] d q^{\prime},
\end{aligned}
$$

получим, изменив нумерацию переменных,

$$
\begin{aligned}
C_{3}(1,2,3)= & \int\left[h_{3}(4,5,6)-h_{2}(4,5) h_{2}(4,6)-h_{2}(4,5) h_{2}(5,6)\right] \times \\
& \times \prod\left[\delta(i, i+3)-C_{2}(i, i+3) \rho(i+3)\right] d(4,5,6)-C_{2}(1,3) C_{2}(2,3)= \\
= & \int\left[h_{3}(4,5,6)-h_{2}(4,5) h_{2}(4,6)-h_{2}(4,5) h_{2}(5,6)-h_{2}(4,6) h_{2}(5,6)\right] \times \\
& \times \prod\left[\delta(i, i+3)-C_{2}(i, i+3) \rho(i+3)\right] d(4,5,6)- \\
& -\int C_{2}(1, q) C_{2}(2, q) C_{2}(3, q) \rho(q) d q .
\end{aligned}
$$


Выражения для $C_{n}$ при $n>3$ можно получить очевидным, но весьма громоздким варьированием последнего выражения.

Из полученного выражения следует также условие выполнимости равенства

$$
1+\rho^{2} \int C_{3}(1,2,3) d(2,3)=0
$$

в критической точке. Имеем

$$
\begin{aligned}
1+ & \rho^{2} \int C_{3}(1,2,3) d(2,3)=\rho^{2}\left(1-\rho \int C_{2}(1,2) d(2)\right)^{3} \int\left[h_{3}(1,2,3)-\right. \\
& \left.-\sum h_{2}(i, j) h_{2}(i, k)\right] d(2,3)+1-\left(\rho \int C_{2}(1,2) d(2)\right)^{3} .
\end{aligned}
$$

Следовательно, интеграл от $h_{3}$ должен расходиться не сильнее (по каждому из двух расстояний), чем интеграл от $h_{2}$.

Благодарности. Работа выполнена при поддержке Минобразования России, грант УР 01.01.002.

\section{Список литературы}

[1] Р. Балеску. Равновесная и неравновесная статистическая механика. Т. 1. М.: Мир, 1978.

[2] Дж. Рашбрук. Равновесные теории жидкого состояния. В сб.: Физика простых жидкостей. Ред. Г. Темперли, Дж. Роулинсон, Дж. Рашбрук. Т. 1. М.: Мир, 1971. С. 30-62.

[3] ШI. Ма. Современная теория критических явлений. М.: Мир, 1980.

[4] Э. А. Аринштейн. Функциональные преобразования в теории частичных функций распределения. В сб.: Проблемы статистической физики. Тр. Тюменского гос. ун-та. Ред. Э. А. Аринштейн. Тюмень: ТюмГУ, 1976. С. 25-50.

[5] Б. Г. Абросимов, Э. А. Аринштейн. Журн. структ. химии. 1969. Т. 10. № 2. С. 320-326.

[6] Э. А. Аринштейн. ДАН СССР. 1969. Т. 189. № 5. С. 502-505.

[7] Э. А. Аринштейн. Соотношение Орнштейна-Цернике в критической области. В сб.: Физика жидкого состояния. Ред. А. С. Кривошея. Киев: Лыбидь, 1990. № 18. С. 121-128.

[8] Н.Я. Виленкин. Специальные функции и теория представлений групп. М.: Наука, 1965.

[9] Г. Бейтман, А. Эрдейн. Таблица интегральных преобразований. Т. 2. М.: Наука, 1970. 